\title{
Combined everolimus and endocrine therapy in advanced HR-positive, HER2-negative Chinese breast cancer patients: a retrospective study
}

\author{
Xia-Bo Shen ${ }^{1,2}$, Guang-Liang $\mathrm{Li}^{1,2}$, Ya-Bing Zheng ${ }^{1,2}$, Zhan-Hong Chen ${ }^{1,2}$, Wen-Ming Cao ${ }^{1,2}$, \\ Xiao-Jia Wang ${ }^{1,2}$, Xi-Ying Shao ${ }^{1,2}$ \\ ${ }^{1}$ Department of Breast Medical Oncology, Cancer Hospital of the University of Chinese Academy of Sciences (Zhejiang Cancer Hospital), \\ Hangzhou, China; ${ }^{2}$ Institute of Cancer and Basic Medicine (IBMC), Chinese Academy of Sciences, Hangzhou, China \\ Contributions: (I) Conception and design: XJ Wang, XY Shao; (II) Administrative support: XJ Wang; (III) Provision of study materials or patients: YB \\ Zheng, ZH Chen, WM Cao; (IV) Collection and assembly of data: YB Zheng, ZH Chen, WM Cao; (V) Data analysis and interpretation: XB Shen, \\ GL Li; (VI) Manuscript writing: All authors; (VII) Final approval of manuscript: All authors. \\ Correspondence to: Xi-Ying Shao; Xiao-Jia Wang. Department of Breast Medical Oncology, Cancer Hospital of the University of Chinese Academy of \\ Sciences (Zhejiang Cancer Hospital), Hangzhou 310022, China. Email: shaoxy@zjcc.org.cn; wxiaojia0803@163.com.
}

Background: Everolimus (EVE) is an inhibitor of the mammalian target of rapamycin (mTOR) pathway, and it is approved for the treatment of advanced breast cancer (ABC). However, there is still little realworld data on using EVE in Chinese breast cancer patients. We retrospectively analyzed real-world data to determine the factors affecting EVE treatment efficacy and patient outcomes.

Methods: We retrospectively collected the treatment information of ABC patients treated with EVE from 2013 to 2020 in Zhejiang Cancer Hospital. Kaplan-Meier analysis and Cox regression methods were used to calculate and compare the progression-free survival (PFS), and identify the factors associated with EVE treatment efficacy.

Results: The study finally enrolled 84 patients meeting the requirement; the median PFS in all 84 patients was 6.87 months. Multivariate analysis showed that liver metastasis [hazard ratio, 1.69; 95\% confidence interval (CI), 1.00-2.84; $\mathrm{P}=0.049$ ], and brain metastasis (hazard ratio, 2.65; 95\% CI, 1.07-6.58; $\mathrm{P}=0.036$ ) were independent risk factors. Subgroup analyses demonstrated EVE + fulvestrant (FUL) was not superior to $\mathrm{EVE}$ + aromatase inhibitors (AIs) for PFS (5.77 vs. 7.97 months, $\mathrm{P}=0.0735$ ). Furthermore, it showed EVE + AI was superior to EVE + FUL in some subgroups: postmenopausal group (hazard ratio, 0.50; 95\% CI, 0.26-0.98); without bone metastasis group (hazard ratio, 0.22; 95\% CI, 0.06-0.80); visceral disease group (hazard ratio, 0.37; 95\% CI, 0.20-0.69).

Conclusions: EVE combined with endocrine therapy is an effective treatment option for Chinese patients with hormone-receptor-positive (HR+), human epidermal growth factor receptor-2-negative (HER2-) breast cancer, although EVE + FUL was not superior to EVE + AI. Liver metastasis and brain metastasis were independent risk factors for successful EVE + endocrine therapy.

Keywords: Advanced breast cancer (ABC); aromatase inhibitors (AIs); everolimus (EVE); fulvestrant (FUL); retrospective study

Submitted Jul 02, 2021. Accepted for publication Aug 12, 2021.

doi: $10.21037 / \mathrm{atm}-21-3840$

View this article at: https://dx.doi.org/10.21037/atm-21-3840

$\wedge$ ORCID: 0000-0002-1643-3842. 


\section{Introduction}

Breast cancer is one of the most important malignant tumors that threaten the health of women worldwide. China is one of the countries with the fastest growth rate of breast cancer, and it ranks first among female malignant tumors, so the burden of cancer is increasing (1). Hormone-receptorpositive $(\mathrm{HR}+)$, human epidermal growth factor receptor2-negative (HER2-) breast cancer is a major subtype and the main treatments for advanced HR+, HER2- breast cancer patients are chemotherapy and endocrine therapy. In particular, endocrine therapy has the advantages of mild and transient side effects, excellent tolerability, and long tumor control time, which is an important aspect for patients with advanced breast cancer (ABC). However, drug resistance and subsequent treatment after endocrine therapy are of clinical concern (2).

Everolimus (EVE) is a mammalian target of rapamycin (mTOR) inhibitor, mainly acting on the phosphatidylinositol 3-kinase (PI3K)-protein kinase B (AKT)-mTOR pathway (3). The Food and Drug Administration approves the combination of EVE and exemestane for the treatment of ABC patients who are HR+, HER2-. Hyperactivation of the PI3K-AKT-mTOR signaling pathway is common in ABC and is implicated in resistance to endocrine therapy $(4,5)$.

However, there is still little real-world data on the use of EVE in Chinese breast cancer patients, and predictors of treatment efficacy remain unclear. It reported that the single proteins or heatmap subgroups of the differentially activated PI3K pathways were unable to discriminate patients on EVE with poor or better prognosis (6). Because the efficacy of EVE is also largely independent of the most commonly altered genes or pathways in breast cancer, the potential impact of chromosomal instabilities and lowfrequency genetic alterations on EVE efficacy remains to be further investigated (7).

Thus, the purpose of this study was to retrospectively analyze real-world data, determine the factors that influence EVE treatment efficacy, and search for a better treatment regimen. We present the following article in accordance with the STROBE reporting checklist (available at https:// dx.doi.org/10.21037/atm-21-3840).

\section{Methods}

\section{Patients}

We enrolled ABC patients treated with EVE from 2013 to 2020 in Zhejiang Cancer Hospital who met the following criteria: $\mathrm{ABC}$ confirmed by pathological or imaging examination; latest pathology testing of the metastatic tumor showed estrogen receptor-positive (ER+), or progesterone receptor-positive $(\mathrm{PR}+)$, HER2- breast cancer; patients received $\mathrm{EVE}$ + endocrine therapy including aromatase inhibitor (AI) or fulvestrant (FUL), not combined with chemotherapy or targeted therapy; patients had detailed medical records and imaging data, and survival follow-up information was available. The flowchart of this retrospective study is shown in Figure 1. This study was approved by the Zhejiang Cancer Hospital Ethics Committee (No. IRB2021-85), and the need for written informed consent was waived by the Ethics Committee because of the retrospective nature. This study was conducted following the Declaration of Helsinki (as revised in 2013).

\section{Data collection}

Data were retrieved from the medical records, including clinical features, history of surgery and adjuvant treatment, organs and number of tumor metastases, the combined EVE treatment regimens, previous treatment lines, dose adjustment, and optimal outcomes. We also inquired of patients or their families by telephone to ascertain the survival time, which was not always available from the medical records.

The progression-free survival (PFS), as the main endpoint, was defined as the time from randomization to disease progression or death. The overall survival (OS) was defined as the time from randomization until death, and the disease-free survival was defined as the time between randomization and disease recurrence or death.

According to hormone levels before EVE medication, the patients are classified into pre-menopausal + ovarian function suppression (OFS) group and post-menopausal group. According to number of organ metastatic sites, the patients are classified into $\leq 2$ organ metastases group and $\geq 3$ organ metastases group. According to presence of visceral disease before EVE medication, the patients are classified into with visceral disease group and without visceral disease group. According to adjuvant endocrine therapy time less than 2 years to relapse, or advanced first-line endocrine therapy time less than 6 months to disease progress, the patients are classified into Primary and secondary resistance group.

\section{Treatments}

EVE was administered at an initial dose (10 or $5 \mathrm{mg}$ /day) 


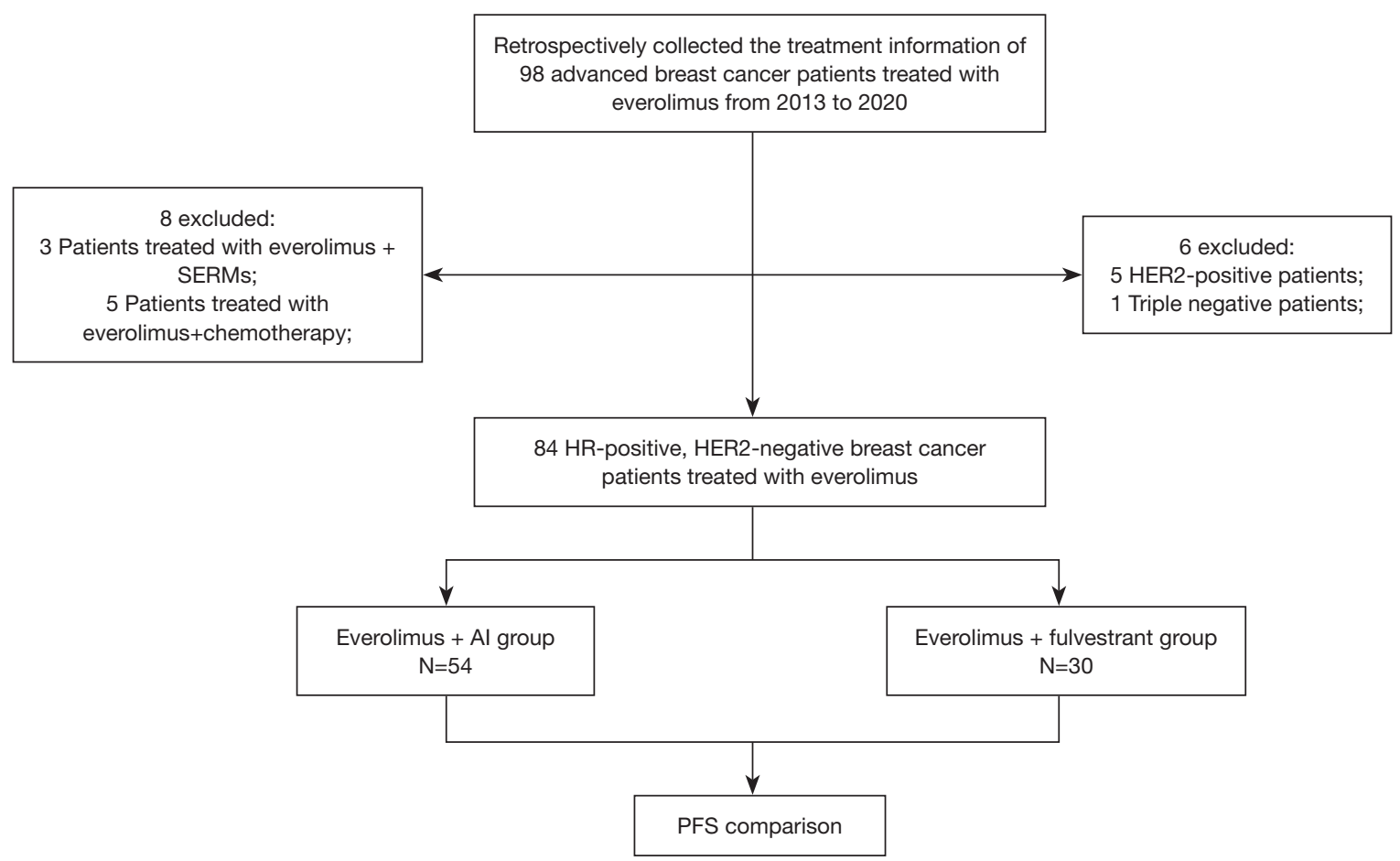

Figure 1 Study flow chart. SERMs, selective estrogen receptor modulators; HER2, human epidermal growth factor receptor-2; HR, hormone-receptor; AI, aromatase inhibitor; PFS, progression-free survival.

and the dose was adjusted ( $5 \mathrm{mg} /$ day) if there were adverse reactions. As for combined therapy regimens, the AI was letrozole $(2.5 \mathrm{mg} /$ day), anastrozole $(1 \mathrm{mg} /$ day $)$, or exemestane ( $25 \mathrm{mg} /$ day). FUL was administered intramuscularly $500 \mathrm{mg}$ on day $1,15,29$, and then every 28 days.

\section{Statistical analysis}

All statistical analysis was performed using SPSS software version 26.0, GraphPad Prism version 8.0 software, and R 3.6.0 software. The chi-squared $\left(\chi^{2}\right)$ test was used to compare rates in two groups. The Kaplan-Meier method was used for survival analysis, and the log-rank test was used for statistical differences. Cox regression analysis was used for univariate and multivariate analysis. A two-sided $\mathrm{P}$ value $<0.05$ was considered statistically significant.

\section{Results}

\section{Clinical characteristics}

The study finally enrolled 84 patients meeting the requirements, the median follow-up time was 14.9 months. The median age was 53.0 (48.0-60.0) years, most patients $(\mathrm{n}=49)$ were postmenopausal and premenopausal patients received OFS treatment. Fifty-five of the enrolled breast cancer patients were ER+ and PR+; there were 80 patients with tumor recurrence after surgical treatment for breast cancer and 4 patients with $\mathrm{ABC}$. The most common sites of tumor metastasis were bone $(\mathrm{n}=65)$, liver $(\mathrm{n}=45)$, lung or pleura $(n=39)$, and regional lymph nodes $(n=35)$. A total of 54 patients received $\mathrm{EVE}+\mathrm{AI}$ and 30 received $\mathrm{EVE}$ + FUL. Moreover, 52 patients previous received AI for advanced diseases before EVE treatment while 45 patients previous received FUL. And 36 patients previous received $<2$ chemotherapy lines for advanced diseases before EVE treatment, 48 patients previous received $<2$ endocrine therapy lines. As for total therapy lines, 36 patients previous received $<4$ therapy lines for advanced diseases before EVE treatment (Table 1).

\section{Efficacy and safety in the overall patient cohort}

The median PFS in all 84 patients was 6.87 months, and the median OS was 28.87 months (Figure 2). The disease 
Table 1 Clinical characteristics of $84 \mathrm{HR}+\mathrm{HER} 2-\mathrm{ABC}$ patients

\begin{tabular}{|c|c|c|}
\hline Clinical characteristics & Cases (n) & $\%$ \\
\hline \multicolumn{3}{|l|}{ Age (years) } \\
\hline$<60$ & 60 & 71.4 \\
\hline$\geq 60$ & 24 & 28.6 \\
\hline Median (IQR) & $53.0(48.0-60.0)$ & \\
\hline \multicolumn{3}{|l|}{ Menopausal status } \\
\hline Premenopausal + OFS & 35 & 41.7 \\
\hline Postmenopausal & 49 & 58.3 \\
\hline \multicolumn{3}{|l|}{ Hormone receptor status } \\
\hline $\mathrm{ER}+, \mathrm{PR}-$ & 27 & 32.1 \\
\hline $\mathrm{ER}-, \mathrm{PR}+$ & 2 & 2.4 \\
\hline $\mathrm{ER}+, \mathrm{PR}+$ & 55 & 65.5 \\
\hline \multicolumn{3}{|l|}{ Metastatic sites } \\
\hline Bone & 65 & 77.4 \\
\hline Liver & 45 & 53.6 \\
\hline Lung or pleura & 39 & 46.4 \\
\hline Regional lymph nodes & 35 & 41.7 \\
\hline Brain & 9 & 10.7 \\
\hline Chest wall & 9 & 10.7 \\
\hline \multicolumn{3}{|c|}{ No. of organ metastatic sites } \\
\hline$\leq 2$ & 63 & 75.0 \\
\hline$\geq 3$ & 21 & 25.0 \\
\hline \multicolumn{3}{|l|}{ Visceral disease } \\
\hline Yes & 65 & 77.4 \\
\hline No & 19 & 22.6 \\
\hline \multicolumn{3}{|l|}{ EVE treatment regimen } \\
\hline $\mathrm{EVE}+\mathrm{Al}$ & 54 & 64.3 \\
\hline EVE + FUL & 30 & 35.7 \\
\hline \multicolumn{3}{|l|}{ EVE dose } \\
\hline $5 \mathrm{mg} / \mathrm{day}$ & 48 & 57.1 \\
\hline $10 \mathrm{mg} /$ day & 36 & 42.9 \\
\hline \multicolumn{3}{|c|}{ Dose adjustment (in 3610 mg/day patients) } \\
\hline No & 21 & 58.3 \\
\hline Yes & 15 & 41.7 \\
\hline
\end{tabular}

Table 1 (continued)
Table 1 (continued)

\begin{tabular}{lcc}
\hline Clinical characteristics & Cases (n) & $\%$ \\
\hline Previous advanced endocrine therapy & 52 & 61.9 \\
Al & 45 & 52.6 \\
FUL & & \\
Previous chemotherapy lines & 36 & 42.9 \\
$<2$ & 48 & 57.1 \\
$\geq 2$ & & \\
Previous endocrine therapy & & \\
lines & 48 & 57.1 \\
$<2$ & 36 & 42.9 \\
$\geq 2$ & & \\
Previous therapy lines & 36 & 52.9 \\
$<4$ & 48 & 57.1 \\
$\geq 4$ &
\end{tabular}

HR, hormone-receptor; HER2, human epidermal growth factor receptor-2; $A B C$, advanced breast cancer; IQR, interquartile range; OFS, ovarian function suppression; $E R$, estrogen receptor; PR, progesterone receptor; EVE, everolimus; AI, aromatase inhibitor; FUL, fulvestrant.

control rate (DCR) in all 84 patients was $76.2 \%$ (Table 2), and the most common grade 3-4 adverse event was stomatitis $(8.3 \%)$, which is similar to other previous studies (Table 3).

\section{PFS comparison for different clinical characteristics and treatment regimens}

Kaplan-Meier analysis showed no statistical difference in PFS between the menopausal status group $(\mathrm{P}=0.2010)$, the visceral disease group $(\mathrm{P}=0.6518)$, the number of organ metastatic sites group $(\mathrm{P}=0.1220)$, or the endocrine therapy resistance group $(\mathrm{P}=0.3885)$ (Figure 3$)$. As for treatment regimens, there was no statistical difference in the PFS between the previous AI/FUL group $(\mathrm{P}=0.6785$ and $\mathrm{P}=0.0525)$. Meanwhile, the dose of EVE had little effect on PFS: the $5 \mathrm{mg}$ /day group had a very similar PFS to the $10 \mathrm{mg}$ /day group [6.80 vs. 6.87 months; hazard ratio, 0.93; 95\% confidence interval (CI), 0.57-1.52; $\mathrm{P}=0.7795]$. The median PFS of patients who had dose de-escalation from 10 
A

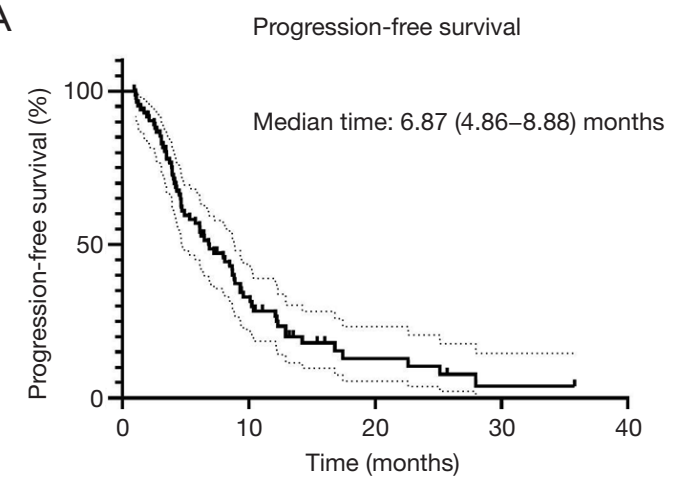

B

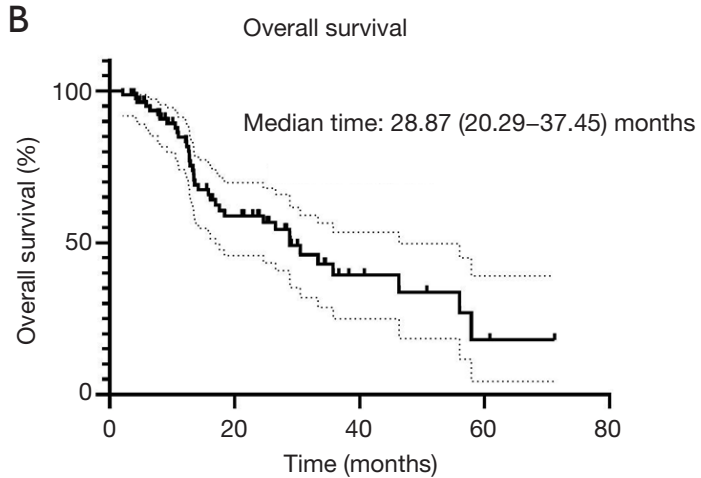

Figure 2 PFS (A) and OS (B) of 84 patients treated with EVE. PFS, progression-free survival; OS, overall survival; EVE, everolimus.

Table 2 Treatment response

\begin{tabular}{lcc}
\hline Best treatment evaluation & Cases $(\mathrm{n})$ & $\%$ \\
\hline Complete response & 0 & 0.0 \\
Partial response & 11 & 13.1 \\
Stable disease & 53 & 63.1 \\
Progressive disease & 20 & 23.8 \\
\hline
\end{tabular}

Table 3 Grade 3-4 adverse events

\begin{tabular}{lcc}
\hline Grade 3-4 adverse event & Cases $(\mathrm{n})$ & $\%$ \\
\hline Stomatitis & 7 & 8.3 \\
ALT/AST increased & 6 & 7.1 \\
Anemia & 4 & 4.8 \\
Hyperglycemia & 3 & 3.6 \\
Hypercholesterolemia & 2 & 2.4 \\
Thrombocytopenia & 2 & 2.4 \\
Fatigue & 2 & 2.4 \\
\hline
\end{tabular}

ALT, alanine aminotransferase; AST, aspartate aminotransferase.

to $5 \mathrm{mg}$ was 8.10 months, while the PFS of patients who did not was 6.13 months $(\mathrm{P}=0.4377)$ (Figure 4).

\section{Univariate and multivariate analyses of PFS}

In all 84 patients, univariate analysis identified age, liver metastasis, brain metastasis, EVE treatment regimen, and previous chemotherapy lines to be associated with PFS $(\mathrm{P}<0.20)$. Multivariate analysis showed that liver metastasis (hazard ratio, 1.69; 95\% CI, 1.00-2.84, $\mathrm{P}=0.049$ ), and brain metastasis (hazard ratio, 2.65; 95\% CI, 1.07-6.58; $\mathrm{P}=0.036)$ were independent risk factors, predicting shorter PFS (Table 4).

\section{Subgroup analyses comparing EVE + AI/FUL}

Subgroup analyses were further performed based on EVE + AI/FUL. Patients in the two groups were well balanced in terms of clinical characteristics (Table 5). The EVE + FUL group showed no superior outcome to the EVE + AI group for PFS (5.77 vs. 7.97 months; hazard ratio, 1.56; 95\% CI, 0.92-2.65; $\mathrm{P}=0.0735)$. Among patients who previously received FUL treatment, the EVE + AI group also showed longer PFS than the EVE + FUL group (7.23 vs. 4.47 months; hazard ratio, $1.73 ; 95 \%$ CI, 0.83-3.61; $\mathrm{P}=0.0998)$. Moreover, in the EVE + FUL group, patients with visceral disease had shorter PFS than those without visceral disease (Figure 5).

Further subgroup analyses showed EVE + AI was superior to EVE + FUL in some subgroups: postmenopausal group (hazard ratio, 0.50 ; $95 \% \mathrm{CI}, 0.26-0.98$ ); without bone metastasis group (hazard ratio, 0.22; 95\% CI, 0.06-0.80); lung or pleura metastasis group (hazard ratio, $0.35 ; 95 \%$ CI, 0.16-0.77); visceral disease group (hazard ratio, $0.37 ; 95 \%$ CI, 0.20-0.69); previous therapy lines $\geq 4$ group (hazard ratio, 0.44; 95\% CI, 0.22-0.87) (Figure 6).

\section{Discussion}

Although cyclin-dependent kinases (CDK) 4/6 inhibitors and histone deacetylase (HDAC) inhibitors had achieved very good results as endocrine treatments of $\mathrm{ABC}(8,9)$, they are poorly available for Chinese patients due to their high price. Therefore, combined EVE therapy is a viable choice 
A

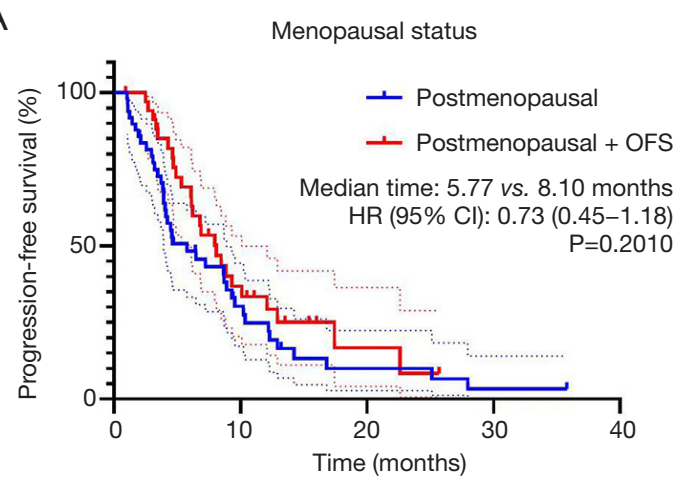

C

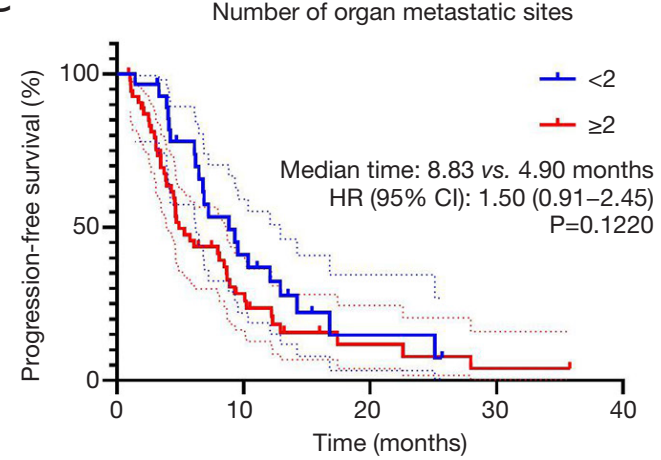

B

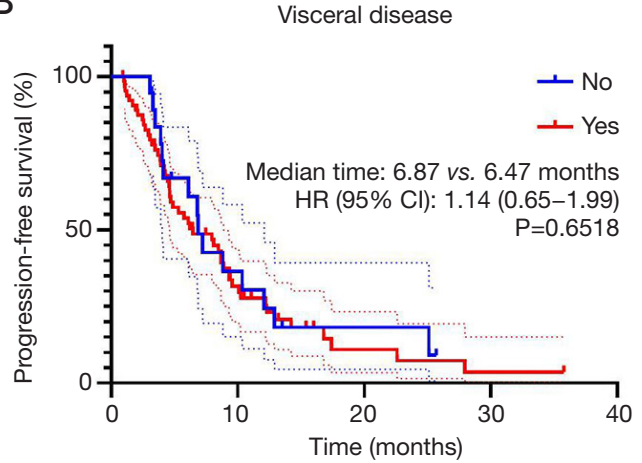

D

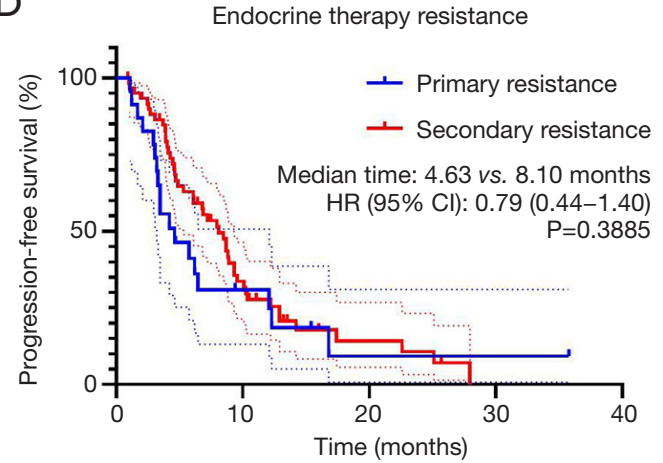

Figure 3 PFS comparison with clinical characteristics: (A) menopausal status; (B) visceral disease; (C) number of organ metastatic sites; (D) endocrine therapy resistance. PFS, progression-free survival; OFS, ovarian function suppression; HR, hazard ratio; CI, confidence interval.

for advanced HR+, HER2- breast cancer patients, although there is scant information on efficacy in Chinese patients.

This study demonstrated that EVE combined with AI or FUL is an effective treatment option with good tolerance. The classic study of EVE was the BOLERO-2 clinical trials, which evaluated the efficacy and safety of the combination of EVE and exemestane in HR+ breast cancer patients refractory to nonsteroidal AI. The results showed the median PFS assessed by the local investigators was 6.9 months for EVE + exemestane, vs. 10.6 months by central assessment (10). As for OS analysis, the median duration was 31.0 months, and the EVE group did not have a significantly reduced risk of death compared with the placebo group (11). Further ethnicity subgroup analysis resulted in a median PFS duration among EVE-treated Asian patients of 8.48 months, and the DCR was $75 \%$, and 7.33 months in non-Asian patients (12). Another realworld retrospective study conducted in China to evaluate the efficacy and safety of EVE + endocrine therapy showed a median PFS of 5.9 months (95\% CI, 4.6-7.2), and the median OS was not reached (13). Safety results were in line with the prior safety profile of the EVE study; the most common grade 3-4 adverse event was stomatitis, and the incidence was around $10 \%$ (14).

The results from this study suggested no difference in PFS for the different menopausal status, visceral disease, drug resistance, EVE dose, and dose adjustment groups. The BOLERO-2 trial's age subgroup analysis showed that the addition of EVE to exemestane increased the median PFS to 6.77 months in elderly patients, and to 8.11 months in younger patients (15). An Austrian study (16) similarly found the median PFS was numerically longer in the $10 \mathrm{mg}$ EVE subgroup compared with the $5 \mathrm{mg}$ EVE subgroup (9.9 vs. 8.0 months). For patients who had dose escalation from 5 to $10 \mathrm{mg}$, the median PFS was 7.8 months while patients who had dose de-escalation from 10 to $5 \mathrm{mg}$, the median PFS was 9.5 months; however, the difference was not statistically significant (16). The MIRACLE study was a clinical trial comparing letrozole and letrozole combined with EVE as a first-line treatment of Chinese patients with HR+/HER2- breast cancer recurrence or metastasis after treatment with tamoxifen. The results showed the 
A
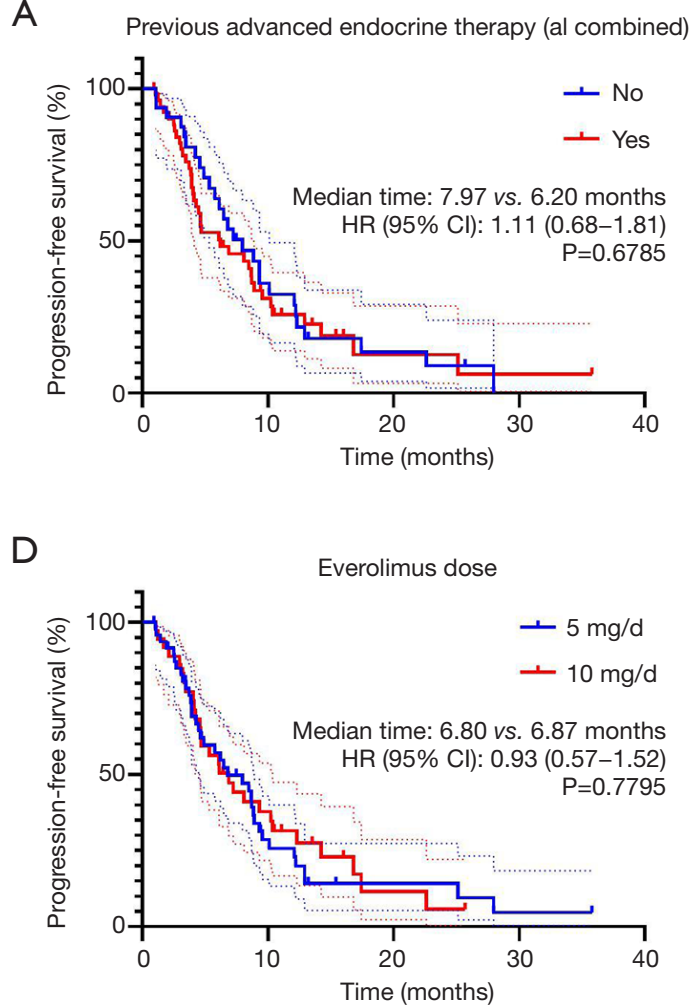

B

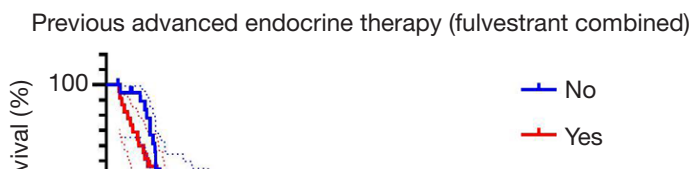
HR $(95 \% \mathrm{Cl}): \begin{array}{r}1.60(0.98-2.61) \\ \mathrm{P}=0.0525\end{array}$

D

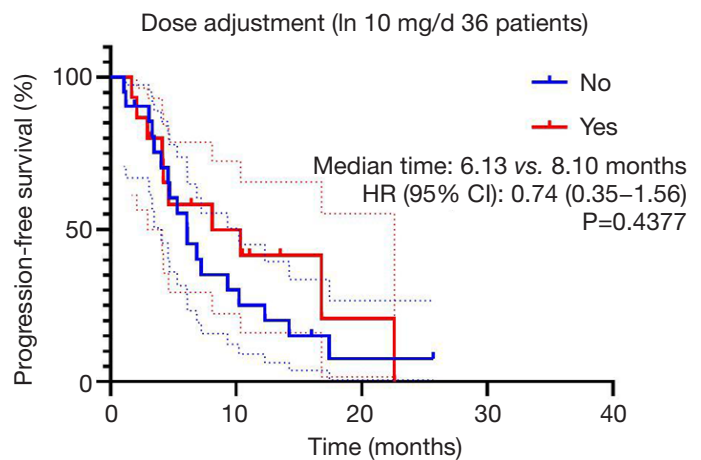

Figure 4 PFS comparison with treatment: (A) previous AI combined therapy; (B) previous FUL combined therapy; (C) different EVE dose; (D) dose adjustment in $3610 \mathrm{mg}$ /day patients. PFS, progression-free survival; AI, aromatase inhibitor; FUL, fulvestrant; EVE, everolimus; HR, hazard ratio; CI, confidence interval.

PFS was 19.2 months, and in the subgroup analysis, PFS benefited from EVE treatment whether there was primary or secondary endocrine resistance (17).

Previous studies have mostly focused on postmenopausal patients. The 4EVER trial evaluated the combination of $\mathrm{EVE}$ and exemestane in postmenopausal women with $\mathrm{HR}+$, HER2 - locally advanced or ABC who had received prior exemestane, FUL, and/or tamoxifen and any number of prior chemotherapy lines. The median PFS was 5.6 months and the median OS time was not reached (18). For postmenopausal breast cancer patients resistant to AIs, another study demonstrated EVE combined with exemestane had a numerical increase compared with FUL, (4.0 vs. 6.1 months, respectively; $\mathrm{P}=0.419$ ) (19). The results of our study showed that in premenopausal patients, EVE also had good efficacy: the PFS of premenopausal patients with ovarian function inhibition was 8.10 vs. 5.77 months in postmenopausal patients.

This study innovatively found that brain metastasis and liver metastasis were independent risk factors for EVE treatment. Though EVE is an mTOR inhibitor, whether mutation of the PI3K-AKT-mTOR signaling pathway is a prognostic factor remains controversial. Sirico et al. found a PET-based biomarker provided additional information on which patients were most likely to benefit from EVE + exemestane-based therapy (20). The EverExt study demonstrated that more patients in the lowest fasting glucose (FG) category achieved clinical benefit compared with the highest group of breast cancer patients treated with EVE + exemestane, and FG at re-assessment was also predictive of PFS. More than that, treatment discontinuation was significantly associated with changes in FG (21). However, this study did not find a correlation between treatment lines and PFS. The BOLERO-4 clinical trial found a median PFS of 22.0 months (95\% CI, 18.125.1 months) with EVE and letrozole as first-line treatment vs. 3.7 months (95\% CI, 1.9-7.4 months) with EVE and exemestane as second-line treatment (22). The PFS gap between BOLERO-4 clinical trial and our study may be that most of the patients we included had undergone multi- 
Table 4 Univariate and multivariate analyses of PFS

\begin{tabular}{|c|c|c|c|c|}
\hline Clinical characteristics & \multicolumn{2}{|c|}{ Univariate analysis } & \multicolumn{2}{|c|}{ Multivariate analysis } \\
\hline Age (year) & & 0.129 & & 0.131 \\
\hline$<60$ & Reference & & Reference & \\
\hline$\geq 60$ & $1.50(0.89-2.53)$ & & $1.52(0.88-2.62)$ & \\
\hline Premenopausal + OFS & Reference & & & \\
\hline Postmenopausal & $1.39(0.84-2.29)$ & & & \\
\hline Bone metastasis & & 0.239 & & \\
\hline No & Reference & & & \\
\hline No & Reference & & Reference & \\
\hline Yes & $1.63(0.98-2.71)$ & & $1.69(1.00-2.84)$ & \\
\hline Lung or pleura metastasis & & 0.649 & & \\
\hline No & Reference & & & \\
\hline Yes & $0.89(0.54-1.46)$ & & & \\
\hline Brain metastasis & & 0.080 & & 0.036 \\
\hline No & Reference & & Reference & \\
\hline Yes & $2.15(0.91-5.07)$ & & $2.65(1.07-6.58)$ & \\
\hline $\mathrm{EVE}+\mathrm{FUL}$ & $1.58(0.95-2.61)$ & & $1.65(0.98-2.79)$ & \\
\hline Previous chemotherapy lines & & 0.155 & & 0.593 \\
\hline$<2$ & Reference & & Reference & \\
\hline$\geq 2$ & $1.47(0.87-2.48)$ & & $1.16(0.67-2.02)$ & \\
\hline Previous endocrine therapy lines & & 0.443 & & \\
\hline$<2$ & Reference & & & \\
\hline$\geq 2$ & $1.22(0.73-2.03)$ & & & \\
\hline
\end{tabular}

PFS, progression-free survival; HR, hazard ratio; Cl, confidence interval; OFS, ovarian function suppression; EVE, everolimus; AI, aromatase inhibitor; FUL, fulvestrant. 
Table 5 Clinical characteristics of the two treatment groups

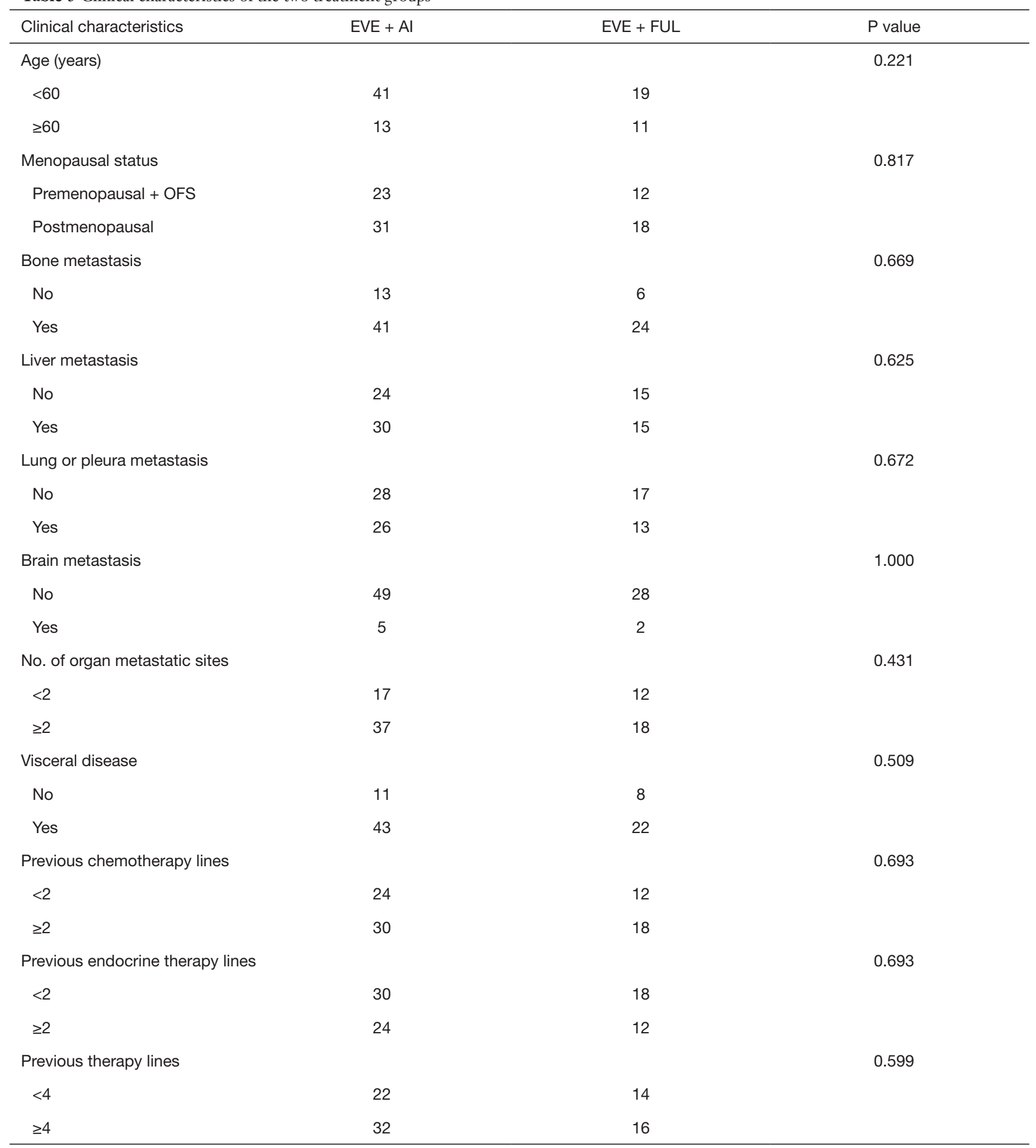

EVE, everolimus; AI, aromatase inhibitor; FUL, fulvestrant; OFS, ovarian function suppression. 
A

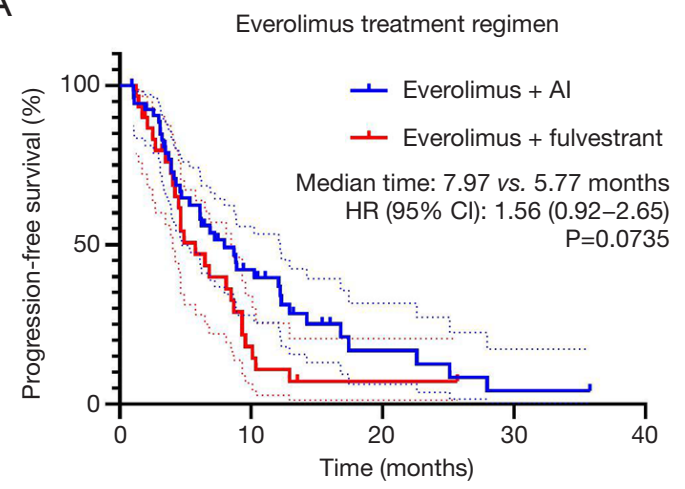

C

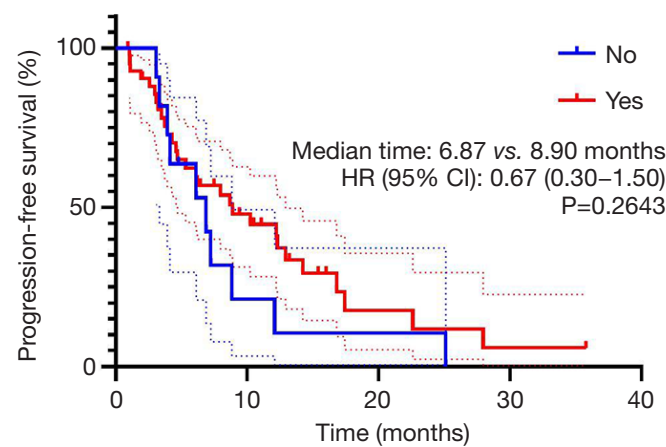

B

Bverolimus treatment regimen (in previous fulvestrant gourp)

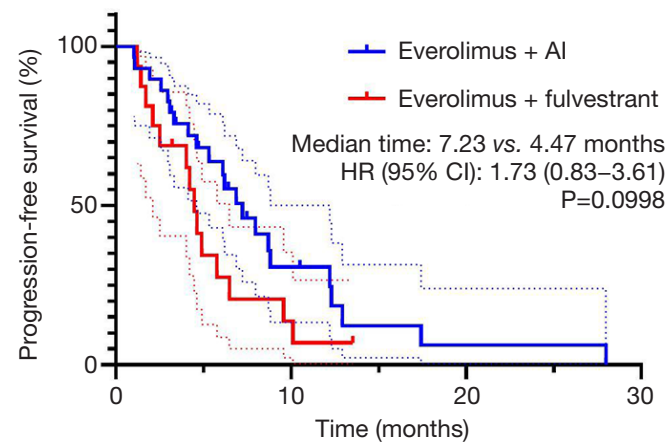

D Visceral disease (in everolimus + fulvestrant group)

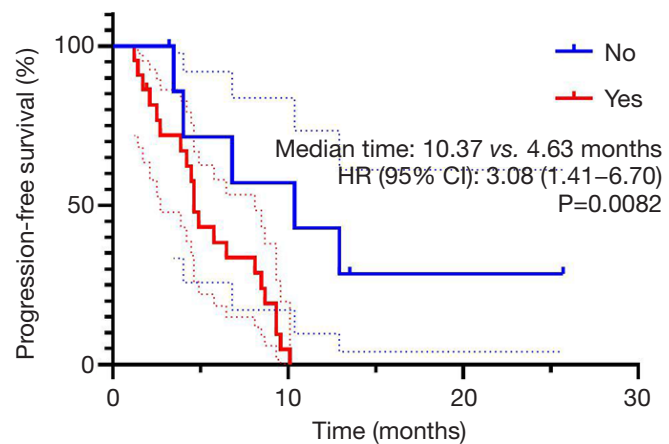

Figure 5 PFS comparisons in subgroups: (A) EVE treatment regimen; (B) previous FUL treatment group (45 patients); (C) EVE + AI group (54 patients); (D) EVE + FUL group (30 patients). PFS, progression-free survival; EVE, everolimus; FUL, fulvestrant; AI, aromatase inhibitor; HR, hazard ratio; CI, confidence interval.

line treatment and could not achieve such a long PFS.

Surprisingly, our subgroup analyses showed that EVE + FUL was not superior to EVE + AI. Moreover, in some groups, EVE + AI showed even better PFS than the EVE + FUL treatment strategy. Previous studies mainly compared $\mathrm{EVE}$ + endocrine therapy to endocrine therapy alone. The PrE0102 study found that EVE + FUL was more efficacious than FUL alone in ER+ ABC resistant to AI therapy. The addition of EVE to FUL improved the median PFS from 5.1 to 10.3 months (23), which was very close to the 10.6 months by central assessment in the BOLERO-2 trial. The FALCON trial also showed a clinical benefit rate of $78 \%$ with FUL and $74 \%$ with anastrozole, which had no statistical difference in $\mathrm{HR}+\mathrm{ABC}$ (24). And during the first 6 months' follow-up, there was no significant difference in PFS between the two groups, which suggested there might not be such a gap between the efficacy of AI and FUL over the short term.

There are some weaknesses in this study. First, some but not all patients had next-generation sequencing tests, so there was insufficient data about the relationship between a PI3K-AKT-mTOR signaling pathway mutation and EVE treatment efficacy. Second, in terms of adverse reactions, we only collected grade 3-4 adverse reactions, because grade 1-2 adverse reactions were partly missing in the electronic medical record. Third, this was a single-center retrospective study, so the outcomes still need further prospective and randomized clinical trials for validation.

\section{Conclusions}

EVE combined with endocrine therapy was an effective treatment option for Chinese patients with HR+, HER2breast cancer. Liver metastasis and brain metastasis were independent risk factors for the efficacy of EVE + endocrine therapy. EVE + FUL was not superior to EVE $+\mathrm{AI}$, but for postmenopausal patients, patients without bone metastasis, and patients with visceral disease, EVE + 


\begin{tabular}{|c|c|c|c|c|c|}
\hline & Events $(n / N)$ & & & & \\
\hline & Everolimus + Al & Everolimus + fulvestrant & & Hazard ratio $(95 \% \mathrm{Cl})$ & $P$ value \\
\hline Age (year) & & & & & 0.084 \\
\hline$<60$ & $28 / 41$ & $16 / 19$ & $-1-1$ & $0.66(0.36-1.24)$ & \\
\hline$\geq 60$ & $11 / 13$ & $10 / 11$ & $\longrightarrow$ & $0.59(0.23-1.46)$ & \\
\hline Menopausal status & & & & & 0.081 \\
\hline Premenopausal + OFS & $16 / 23$ & $9 / 12$ & $\longrightarrow$ & $0.90(0.39-2.03)$ & \\
\hline Premenopausal & $23 / 31$ & $17 / 18$ & 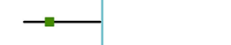 & $0.50(0.26-0.98)$ & \\
\hline Bone metastasis & & & & & 0.070 \\
\hline No & $9 / 13$ & $6 / 6$ & - & $0.22(0.06-0.80)$ & \\
\hline Yes & $30 / 41$ & $20 / 24$ & 一 & $0.76(0.43-1.35)$ & \\
\hline Liver metastasis & & & & & 0.038 \\
\hline No & $15 / 24$ & $12 / 15$ & $\longrightarrow-$ & $0.63(0.29-1.36)$ & \\
\hline Yes & $24 / 30$ & $14 / 15$ & $一-$ & $0.53(0.26-1.10)$ & \\
\hline Lung or pleura metastasis & & & & & 0.086 \\
\hline No & $23 / 28$ & $13 / 17$ & - & $0.99(0.50-1.96)$ & \\
\hline Yes & $16 / 26$ & $13 / 13$ & $\rightarrow-$ & $0.35(0.16-0.77)$ & \\
\hline Brain metastasis & & & & & 0.067 \\
\hline No & $35 / 49$ & $24 / 28$ & $\longrightarrow$ & $0.62(0.36-1.05)$ & \\
\hline Yes & $4 / 5$ & $2 / 2$ & 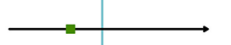 & $0.70(0.12-4.27)$ & \\
\hline Number of organ metastatic sites & & & & & 0.042 \\
\hline$<2$ & $12 / 17$ & $9 / 12$ & $\longrightarrow$ & $0.94(0.39-2.24)$ & \\
\hline$\geq 2$ & $27 / 37$ & $17 / 18$ & - & $0.44(0.23-0.85)$ & \\
\hline Visceral disease & & & & & 0.047 \\
\hline No & $10 / 11$ & $5 / 8$ & & $2.05(0.68-6.14)$ & \\
\hline Yes & $29 / 43$ & $21 / 22$ & $\rightarrow-$ & $0.37(0.20-0.69)$ & \\
\hline Previous chemotherapy lines & & & & & 0.059 \\
\hline$<2$ & $16 / 24$ & $9 / 12$ & - $=$ & $0.57(0.25-1.33)$ & \\
\hline$\geq 2$ & $23 / 30$ & $17 / 18$ & $-1-$ & $0.64(0.33-1.23)$ & \\
\hline Previous endocrine therapy lines & & & & & 0.074 \\
\hline$<2$ & $23 / 30$ & $15 / 18$ & $\longrightarrow-$ & $0.68(0.35-1.34)$ & \\
\hline$\geq 2$ & $16 / 24$ & $11 / 12$ & $\longrightarrow-$ & $0.57(0.26-1.24)$ & \\
\hline Previous therapy lines & & & & & 0.033 \\
\hline$<4$ & $16 / 22$ & $11 / 14$ & $\longrightarrow$ & $0.80(0.36-1.75)$ & \\
\hline$\geq 4$ & $23 / 32$ & $15 / 16$ & - & $0.44(0.22-0.87)$ & \\
\hline Overall & $39 / 54$ & $26 / 30$ & $\longrightarrow$ & $0.63(0.38-1.05)$ & 0.073 \\
\hline & & & $\begin{array}{lllll}0 & 0.5 & 1.0 & 1.5 & 2.0 \\
E v e+A l & & 1.0 v e+F U L\end{array}$ & & \\
\hline
\end{tabular}

Figure 6 Subgroup analyses of the two treatment groups. AI, aromatase inhibitor; CI, confidence interval; OFS, ovarian function suppression; FUL, fulvestrant.

AI was a better choice.

\section{Acknowledgments}

The authors thank all patients enrolled in this study.

Funding: This study was supported by the Zhejiang Provincial Research Center for Cancer Intelligent Diagnosis and Molecular Technology (JBZX-202003).

\section{Footnote}

Reporting Checklist: The authors have completed the STROBE reporting checklist. Available at https://dx.doi. org/10.21037/atm-21-3840

Data Sharing Statement: Available at https://dx.doi. org/10.21037/atm-21-3840 
Conflicts of Interest: All authors have completed the ICMJE uniform disclosure form (available at https://dx.doi. org/10.21037/atm-21-3840). The authors have no conflicts of interest to declare.

Etbical Statement: The authors are accountable for all aspects of the work in ensuring that questions related to the accuracy or integrity of any part of the work are appropriately investigated and resolved. This study was approved by the Zhejiang Cancer Hospital Ethics Committee (No. IRB-2021-85), and the need for written informed consent was waived by the Ethics Committee because of the retrospective nature. This study was conducted following the Declaration of Helsinki (as revised in 2013).

Open Access Statement: This is an Open Access article distributed in accordance with the Creative Commons Attribution-NonCommercial-NoDerivs 4.0 International License (CC BY-NC-ND 4.0), which permits the noncommercial replication and distribution of the article with the strict proviso that no changes or edits are made and the original work is properly cited (including links to both the formal publication through the relevant DOI and the license). See: https://creativecommons.org/licenses/by-nc-nd/4.0/.

\section{References}

1. Chen $\mathrm{W}$, Zheng R, Baade PD, et al. Cancer statistics in China, 2015. CA Cancer J Clin 2016;66:115-32.

2. du Rusquec P, Blonz C, Frenel JS, et al. Targeting the $\mathrm{PI} 3 \mathrm{~K} / \mathrm{Akt} / \mathrm{mTOR}$ pathway in estrogen-receptor positive HER2 negative advanced breast cancer. Ther Adv Med Oncol 2020;12:1758835920940939.

3. Sharma M, Duan Z, Zhao H, et al. Real-world patterns of everolimus use in patients with metastatic breast cancer. Oncologist 2020;25:937-42.

4. Kaklamani VG, Richardson AL, Arteaga CL. Exploring biomarkers of phosphoinositide 3-kinase pathway activation in the treatment of hormone receptor positive, human epidermal growth receptor 2 negative advanced breast cancer. Oncologist 2019;24:305-12.

5. O'Shaughnessy J, Thaddeus Beck J, Royce M. Everolimusbased combination therapies for HR+, HER2- metastatic breast cancer. Cancer Treat Rev 2018;69:204-14.

6. Kruger DT, Opdam M, van der Noort V, et al. PI3K pathway protein analyses in metastatic breast cancer patients receiving standard everolimus and exemestane. J
Cancer Res Clin Oncol 2020;146:3013-23.

7. Hortobagyi GN, Chen D, Piccart M, et al. Correlative analysis of genetic alterations and everolimus benefit in hormone receptor-positive, human epidermal growth factor receptor 2-negative advanced breast cancer: results from BOLERO-2. J Clin Oncol 2016;34:419-26.

8. Cristofanilli M, Turner NC, Bondarenko I, et al. Fulvestrant plus palbociclib versus fulvestrant plus placebo for treatment of hormone-receptor-positive, HER2negative metastatic breast cancer that progressed on previous endocrine therapy (PALOMA-3): final analysis of the multicentre, double-blind, phase 3 randomised controlled trial. Lancet Oncol 2016;17:425-39.

9. Jiang Z, Li W, Hu X, et al. Tucidinostat plus exemestane for postmenopausal patients with advanced, hormone receptor-positive breast cancer (ACE): a randomised, double-blind, placebo-controlled, phase 3 trial. Lancet Oncol 2019;20:806-15.

10. Baselga J, Campone M, Piccart M, et al. Everolimus in postmenopausal hormone-receptor-positive advanced breast cancer. N Engl J Med 2012;366:520-9.

11. Piccart M, Hortobagyi GN, Campone M, et al. Everolimus plus exemestane for hormone-receptor-positive, human epidermal growth factor receptor-2-negative advanced breast cancer: overall survival results from BOLERO-2†. Ann Oncol 2014;25:2357-62.

12. Noguchi S, Masuda N, Iwata H, et al. Efficacy of everolimus with exemestane versus exemestane alone in Asian patients with HER2-negative, hormone-receptorpositive breast cancer in BOLERO-2. Breast Cancer 2014;21:703-14.

13. Gong C, Zhao Y, Wang B, et al. Efficacy and safety of everolimus in Chinese metastatic HR positive, HER2 negative breast cancer patients: a real-world retrospective study. Oncotarget 2017;8:59810-22.

14. Jerusalem G, Mariani G, Ciruelos EM, et al. Safety of everolimus plus exemestane in patients with hormonereceptor-positive, HER2-negative locally advanced or metastatic breast cancer progressing on prior non-steroidal aromatase inhibitors: primary results of a phase IIIb, open-label, single-arm, expanded-access multicenter trial (BALLET). Ann Oncol 2016;27:1719-25.

15. Pritchard KI, Burris HA 3rd, Ito Y, et al. Safety and efficacy of everolimus with exemestane vs. exemestane alone in elderly patients with HER2-negative, hormone receptor-positive breast cancer in BOLERO-2. Clin Breast Cancer 2013;13:421-32.e8.

16. Steger GG, Egle D, Bartsch R, et al. Efficacy and safety 
of everolimus plus exemestane in patients with $\mathrm{HR}+$, HER2 - advanced breast cancer progressing on/after prior endocrine therapy in routine clinical practice: primary results from the non-interventional study, STEPAUT. Breast 2020;50:64-70.

17. Fan Y, Sun T, Shao Z, et al. Abstract PD7-08: Everolimus in first-line treatment of advanced premenopausal breast cancer: results from MIRACLE (everoliMus trIal foR AdvanCed premenopausaL brEast cancer) study. San Antonio: 2019 San Antonio Breast Cancer Symposium. doi: 10.1158/1538-7445.SABCS19-PD7-08.

18. Tesch H, Stoetzer O, Decker T, et al. Efficacy and safety of everolimus plus exemestane in postmenopausal women with hormone receptor-positive, human epidermal growth factor receptor 2-negative locally advanced or metastatic breast cancer: Results of the single-arm, phase IIIB 4EVER trial. Int J Cancer 2019;144:877-85.

19. Li Y, Xie Y, Gong C, et al. Comparative treatment patterns and outcomes of fulvestrant versus everolimus plus exemestane for postmenopausal metastatic breast cancer resistant to aromatase inhibitors in real-world experience. Ther Clin Risk Manag 2020;16:607-15.

20. Sirico M, Bernocchi O, Sobhani N, et al. Early changes of the standardized uptake values (SUVmax) predict the efficacy of everolimus-exemestane in patients with

Cite this article as: Shen XB, Li GL, Zheng YB, Chen ZH, Cao WM, Wang XJ, Shao XY. Combined everolimus and endocrine therapy in advanced HR-positive, HER2-negative Chinese breast cancer patients: a retrospective study. Ann Transl Med 2021;9(16):1334. doi: 10.21037/atm-21-3840 hormone receptor-positive metastatic breast cancer. Cancers (Basel) 2020;12:3314.

21. Pizzuti L, Marchetti P, Natoli C, et al. Fasting glucose and body mass index as predictors of activity in breast cancer patients treated with everolimus-exemestane: The EverExt study. Sci Rep 2017;7:10597.

22. Royce M, Bachelot T, Villanueva C, et al. Everolimus plus endocrine therapy for postmenopausal women with estrogen receptor-positive, human epidermal growth factor receptor 2-negative advanced breast cancer: a clinical trial. JAMA Oncol 2018;4:977-84.

23. Kornblum N, Zhao F, Manola J, et al. Randomized phase II trial of fulvestrant plus everolimus or placebo in postmenopausal women with hormone receptorpositive, human epidermal growth factor receptor 2-negative metastatic breast cancer resistant to aromatase inhibitor therapy: results of PrE0102. J Clin Oncol 2018;36:1556-63.

24. Robertson JFR, Bondarenko IM, Trishkina E, et al. Fulvestrant $500 \mathrm{mg}$ versus anastrozole $1 \mathrm{mg}$ for hormone receptor-positive advanced breast cancer (FALCON): an international, randomised, double-blind, phase 3 trial. Lancet 2016;388:2997-3005.

(English Language Editor: K. Brown) 